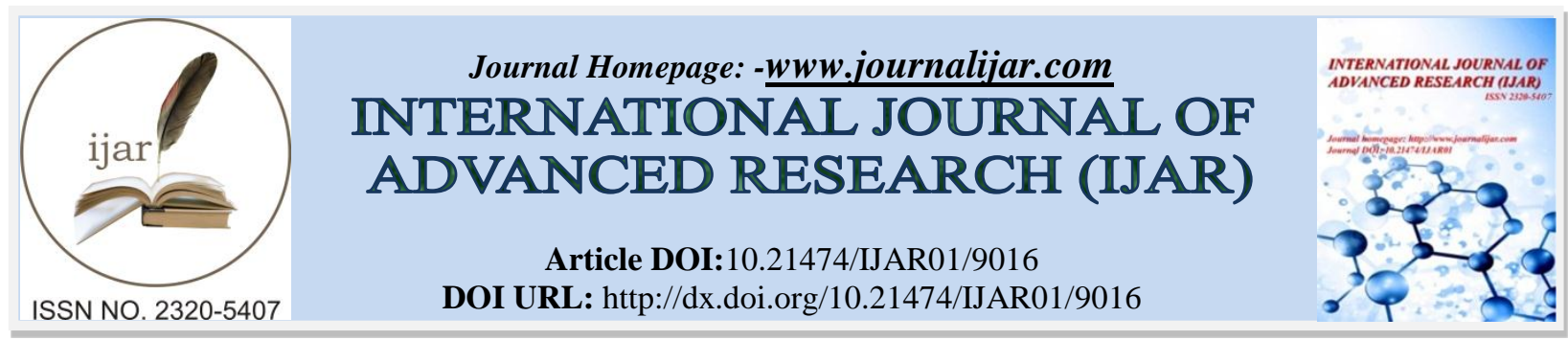

RESEARCH ARTICLE

\title{
A CASE SERIES OF DENGUE HEMORRHAGIC FEVER WITH COMPLICATION, EMPHASIS ON FLUID MANAGEMENT AND COMPLICATION OF RAPID FLUID REPLACEMENT.
}

1. Associate professor, department of medicine kims karad.

2. Junior resident, department of medicine kims karad.

\section{Manuscript Info}

Manuscript History

Received: 04 March 2019

Final Accepted: 06 April 2019

Published: May 2019

\section{Abstract}

Copy Right, IJAR, 2019,. All rights reserved.

\section{Introduction:-}

Dengue viruses (DV) belong to family Flaviviridae and have four serotypes ${ }^{1-4}$. DV are highly adapted to the mosquito and are maintained by vertical transmission. They are transmitted mainly by the Aedes aegypti mosquito and also by Aedes albopictus. It produces from a subclinical infection to a mild self limiting disease, the dengue fever (DF), dengue hemorrhagic fever/ dengue shock syndrome (DHF/DSS).Infection by any one of the four serotypes of dengue virus (DENV) remains asymptomatic in the vast majority.

DHF is most commonly seen in children with secondary dengue infection but has been documented in primary infection with DENV-1 and DENV-3.Hemorrhagic manifestations could be mucosal and or skin or even a positive tourniquet test which is the commonest. Hepatomegaly occurs at some stage of DHF. Abnormal homeostasis and plasma leakage are the main patho-physiological hall marks in DHF. . An estimated 500,000 cases of DHF require hospitalization each year, of whom a very large proportion are children. ${ }^{5,6}$

\section{Case Report-}

Case 1-

A 19 year old male patient admitted to hospital with complaints of fever with chills, generalized weakness and arthralgia. Patient was admitted in wards, after 2 hours of admission patient had 1 episode of epistaxis and followed with massive vomiting containing blood clots in it. Patient was duly transferred over to ICU. All routine lab investigations were sent which were Hb-10.2, TLC-4400, PLATLET COUNT-90000 INR-1.8 BUN-56 CRET-1.6, dengue positive for NS1 antigen and other obvious cause such as Leptospira, Chickenguniya and Malaria were all negative rest of the lab investigation were with in normal limits. Chest $\mathrm{x}$ ray and ECG were also normal. Patient had recurrent epistaxis and vomiting with sustained drop in $\mathrm{HB}$ and platelet count. Pt was given crystalloid fluid at 150 $\mathrm{ml} / \mathrm{hr}$ and pt was transfused with $2 \mathrm{PCV}, 8 \mathrm{RDP}$ and $8 \mathrm{FFP}$. On subsequent days patient become haemodynamically stable and symptomatically better. No antibiotics was given to patients during 7 days of hospital course.

\section{Case 2-}

A 26 year old male patient admitted to hospital with complaints of fever with chills arthralgia and hematemesis. Patient had peculiar erythromatus rash over whole body and blanched when pressed. Ryle's tube was passed and $800 \mathrm{ml}$ of fluid got collected in bag after 15 minutes. All routine lab investigations were send till that patient was given IV fluid crystalloid at $100 \mathrm{ml} / \mathrm{hr}$ and Inj Pantoprazole and Octreotide drip were started. Lab investigations revealed HB-8.3,TLC-7600,PC-15000,INR-2.5,BUN-40,CRT-1.9,SGPT-836,SGOT-690,TOTAL BILLIRUBIN - 
3.6 D-1.1 I-2.5, dengue positive for NS1 antigen and other obvious cause such as Leptospira Chickenguniya and Malaria were all negative. Blood culture was sterile after 7 days of inoculation. Patient was continued on IV fluids and 4 single donor platelets and 2 PCV transfusions were given during hospital stay. Subsequent days patients RT collection reduced and all lab parameters came to with normal limits

\section{Case - 3}

A 65 year old female admitted to hospital with complaints of fever with chills hematemesis and arthralgia. All routine investigation sent with reports were revealed HB-9.6,TLC-4500,PC-30000,INR-1.3,BUN-67,CRT1.1,SGPT-110,SGOT-134,TOTAL BILLIRUBIN -1.6 D-0.8 I-0.8, dengue positive for NS1 antigen and other obvious cause such as Leptospira Chickenguniya and Malaria were all negative. Patient was started on IV fluid with goal being volume replacement. Patient was given 4 RDP along with IVF RL at $60 \mathrm{ml} / \mathrm{hr}$. Patients started complaining of breathlessness at early hours and repeat X RAY was suggestive of pulmonary edema with Congestive Cardiac Failure. Central venous pressure was more than $15 \mathrm{~cm}$. patients fluid was hold and diuretics were started and to maintain BP inotropic support was started. In spite of fluid withdrawal patient started to be symptomatically better and followed by normal lab parameters.

All patients on basis of clinical manifestation and dengue NS1AG positive status were diagnosed as Dengue Hemorrhagic Fever.

\section{Discussion:-}

The dengue viruses are members of the genus Flavivirus and family Flaviviridae. These small $(50 \mathrm{~nm})$ viruses contain single-strand RNA as genome. The virion consists of a nucleocapsid with cubic symmetry enclosed in a lipoprotein envelope. The dengue virus genome is 11644 nucleotides in length, and is composed of three structural protein genes encoding the nucleocapsid or core protein $(\mathrm{C})$, a membrane-associated protein $(\mathrm{M})$, an envelope protein (E), and seven non-structural protein (NS) genes. Among non-structural proteins, envelope glycoprotein, NS1, is of diagnostic and pathological importance. It is $45 \mathrm{kDa}$ in size and associated with viral haemagglutination and neutralization activity. The dengue viruses form a distinct complex within the genus Flavivirus based on antigenic and biological characteristics. There are four virus serotypes, which are designated as DENV-1, DENV-2, DENV-3 and DENV-4. Infection with any one serotype confers lifelong immunity to that virus serotype. Although all four serotypes are antigenically similar, they are different enough to elicit cross-protection for only a few months after infection by any one of them. Secondary infection with another serotype or multiple infections with different serotypes leads to severe form of dengue (DHF/DSS). Aedes (Stegomyia) aegypti (Ae. aegypti) and Aedes (Stegomyia) albopictus (Ae. albopictus) are the two most important vectors of dengue. Aedes (Stegomyia) albopictus belongs to the scutellaris group of subgenus Stegomyia. It is an Asian species indigenous to South East Asia and islands of the Western Pacific and the Indian Ocean. However, during the last few decades the species has spread to Africa, West Asia, Europe and the Americas (North and South) The viraemia among humans builds up high titers two days before the onset of the fever (non-febrile) and lasts 5-7 days after the onset of the fever (febrile). It is only during these two periods that the vector species gets infected.

Transmission of dengue viruses occur in three cycles: Enzootic cycle: A primitive sylvatic cycle maintained by monkey-Aedes-monkey cycle as reported from South Asia and Africa. Viruses are not pathogenic to monkeys and viraemia lasts $2-3$ days. ${ }^{2,3}$ All the four dengue serotypes (DENV-1 to -4 ) have been isolated from monkeys.

Epizootic cycle: The dengue virus crosses over to non-human primates from adjoining human epidemic cycles by bridge vectors. In Sri Lanka, the epizootic cycle was observed among mosquitos (Macaca sinica) during 1986-1987 in a study area on a serological basis. Within the study area (three kilometers), 94\% macaques were found affected.20 Epidemic cycle: The epidemic cycle is maintained by human-Aedes aegypti-human cycle with periodic/cyclical epidemics. Generally, all serotypes circulate and give rise to hyper endemicity. Ae. aegypti has generally low susceptibility to oral infection but its strong anthrophily with multiple feeding behavior and highly domesticated habitats makes it an efficient vector. The persistence of dengue virus, therefore, depends on the development of high viral titers in the human host to ensure transmission in mosquitoes.21Dengue transmission usually occurs during the rainy season when the temperature and humidity are conducive for build-up of the vector population breeding in secondary habitats as well as for longer mosquito survival.

Early diagnosis of dengue virus infection is important and can be established with easily available laboratory tests. Low platelet counts do not predict clinically significant bleeding in dengue. It follows that platelet or blood 
transfusions should not be administered based upon platelet count alone. Bleeding is most likely caused by activated platelets resulting from damaged capillary endothelium

\section{WHO Case Definition of $\mathrm{DHF}^{7}$}

All of the following criteria must be present:

1. Fever (high and continuous of 2-7 days' duration)

2. Hemorrhagic diathesis (at least a positive tourniquet test (except in shock))

3. Thrombocytopenia (less than $100,000 / \mathrm{mm}^{3}$ )

4. Haemoconcentration ( $20 \%$ or more relative to baseline or evidence of increased capillary permeability) or evidence of plasma leakage (i.e. pleural effusion, ascites and/or hypo proteinaemia )

\section{Other clinical manifestations suggestive of DHF are:}

Hepatomegaly (which may be tender),Circulatory disturbances (restlessness, cool extremities, capillary refill time > 2 sec., tachycardia), A fall in hematocrit following volume replacement These along with a platelet count below $100,000 / \mathrm{mm}^{3}$ can justify notification of the case as DHF. Haemoconcentration may be absent during early fluid replacement or in cases where bleeding has occurred.

Dengue virus infections can cause a spectrum of illness ranging from the asymptomatic mild undifferentiated fever, to classical dengue fever (DF), dengue fever with hemorrhagic manifestations, and dengue hemorrhagic fever (DHF)

\section{Criteria for admission (any of the following) in the presence of suspicion of dengue fever:}

1. Restlessness or lethargy

2. Cold extremities or circumoral cyanosis

3. Bleeding in any form

4. Oliguria or reluctance to drink fluids

5. Rapid and weak pulse

6. Capillary refill time $>2$ seconds

7. Narrowing of pulse pressure $(<20 \mathrm{mmHg})$ or hypotension

8. Hematocrit of 40 , or rising hematocrit

9. Platelet count of less than $100,000 / \mathrm{mm}^{3}$

10. Acute abdominal pain

11. Evidence of plasma leakage, e.g. pleural effusion, ascites

\section{Management of Grades I and II DHF-}

1. All cases are to be admitted

2. Encourage patient to drink fluids, ORS, fruit-juices

3. Start intravenous fluid (1/5 dextrose saline initially) for those with poor oral intake.

4. Paracetamol for high fever

5. Daily capillary hematocrit determination (hematocrit will rise before pulse or blood pressure changes)

6. Rise in hematocrit of $20 \%$ or more reflects a significant plasma loss and also indicates need for intravenous fluid therapy

7. Monitor urine output, vital signs

\section{Management of Dengue Shock Syndrome (Grades III and IV DHF)}

This is a medical emergency, and every minute counts towards a favorable outcome. Prompt and adequate fluid replacement for the rapid and massive plasma losses through increased capillary permeability is required. Delayed or inadequate fluid resuscitation can cause multisystem organ dysfunction that may lead to death. Intravenous fluid therapy should be adjusted after close monitoring at 1-2 hourly intervals, throughout the 24-hour period. Establishment of central venous pressure may be necessary in the management of severe cases that are not easily reversible. Colloidal fluid is indicated in patients with massive plasma leakage and in whom a large volume of crystalloid has been given. In cases with persistent shock despite a decline in hematocrit after initial fluid replacement and resuscitation with plasma or plasma expanders, internal bleeding should be suspected. Blood transfusions may then be indicated.

\section{WHO Management Guidelines for DSS}

Infuse $0.9 \%$ saline or Ringer's lactate at $10-20 \mathrm{ml} / \mathrm{kg}$ boluses as rapidly as possible until vital signs return to normal. 2-3 boluses may be needed in profound shock. When vital signs improve, change IV fluids to dextrose 5\% and 
$0.45 \%$ saline at a reduced rate, $1-2 \mathrm{x}$ maintenance $(3-6 \mathrm{ml} / \mathrm{kg} / \mathrm{hour})$, guided by hematocrit, urine output and vital signs. If there is no definite improvement in vital signs and if hematocrit remains high, use plasma volume expander. If there is no definite improvement in vital signs and if hematocrit is low, or has decreased, transfuse blood because this signifies hemorrhage, occult or obvious. Sudden drop in hemoglobin level is also an indicator of occult hemorrhage. Continue replacement of further plasma losses with Dextrose $5 \%$ and $0.45 \%$ saline over a period of 24 48 hours. Reduce or discontinue intravenous fluids between 24-48 hours after the onset of shock if vital signs are stable. Reduce intravenous fluids earlier if patient has good urine output. (Pulmonary edema and massive pleural effusion will occur if excessive intravenous fluids are given after this stage).Hypernatremia and acidosis occur commonly in DSS. These will correct with fluid resuscitation with $0.9 \%$ saline. Periodic arterial blood gases and electrolytes should be measured. Blood transfusion is indicated in significant clinical bleeding, most often hematemesis and melena. Persistent shock with rapidly declining hematocrit level despite adequate volume replacement, indicates significant clinical bleeding which requires prompt treatment with blood transfusion It may be difficult to estimate and recognize the degree of internal blood loss in the presence of haemoconcentration Blood products like fresh frozen plasma, platelet concentrate, and cryoprecipitate may be indicated in some cases, especially when consumptive coagulopathy causes significant bleeding. Platelet concentrate is required if the platelet count is $<50,000 / \mathrm{mm}^{3}$ with bleeding. In the absence of bleeding, prophylactic platelet concentrate is indicated when the platelet count is less than 10,000 to $20,000 / \mathrm{mm}^{3}\left(10-20 \mathrm{ml} / \mathrm{kg}\right.$ of platelets or $\left.4 \mathrm{u} / \mathrm{m}^{2}\right)$.

In the presence of disseminated intravascular coagulation (DIC) ${ }^{8}$, supportive therapy consisting of maintaining circulatory volume, correcting acidosis with sodium bicarbonate and hypoxia with oxygen are required in addition to the use of blood products. Cryoprecipitate ( 1 unit per $5 \mathrm{~kg}$ body weight) followed by platelets $\left(4 \mathrm{units} / \mathrm{m}^{2}\right.$ or $10-20$ $\mathrm{ml} / \mathrm{kg}$ ) within one hour and fresh frozen plasma (FFP - 10-20 ml/kg). Frequent clinical assessment and regular coagulation profile (PT, aPTT, fibrinogen, platelet and FDP) are mandatory as indicated

\section{Criteria for discharging patients hospitalized with dengue/DHF ${ }^{9}$}

All of the following six criteria must be met before a patient is discharged:

1. Absence of fever for 24 hours without the use of antipyretics, and a return of appetite

2. Visible improvement in clinical picture

3. Stable hematocrit

4. Three days after recovery from shock

5. Platelet count greater than $50,000 / \mathrm{mm}^{3}$ and rising

6. No respiratory distress

\section{Conclusion:-}

Dengue fever has spectrum of entity ranging from self resolving infection to life threatening shock. Prompt management is needed with early fluid replacement and if needed blood products should be given. Fluid replacement is dual edged sword as explained in one of the case, patients might land up in fluid overload if excessive fluid intake with prompt monitoring is not done.

\section{Refferances:-}

1. Abhyankar A V, Dash P K, Saxena P, Bhargava R, Parida M M, Jana A M, Sahni A K and Rao P V 2006 Comparison of a dipstick dot-ELISA with commercial assays for anti-dengue virus IgM antibodies; Viral Immunol. 19 630-636

2. Agarwal R, Chaturvedi U C, Misra A, Mukerjee R, Kapoor S, Nagar R, Tandon R and Mathur A 1998 Production of cytotoxic factor by peripheral blood mononuclear cells (PBMC) in patients with dengue haemorrhagic fever; Clin Exp Immunol. 112 340-344

3. Agarwal R, Kapoor S, Nagar R, Misra A, Tandon R, Mathur A, Misra A K, Srivastava K L and Chaturvedi U C 1999a A clinical study of the patients with dengue haemorrhgic fever during the epidemic of 1996 at Lucknow, India, Southeast Asian J. Trop. Med. Publ. Hlth. 30 735-740

4. Agarwal R, Elbishbishi E A, Chaturvedi U C, Nagar R and Mustafa A S 1999b Profi le of transforming growth factor-beta1 in patients with dengue haemorrhagic fever; Int. J. Exp. Pathol. 80 143-149

5. Halstead SB. Dengue. Curr Opin Infect Dis 2002;15:471-476.

6. Chaturvedi UC, Raghupathy R, Pacsa AS, Elbishbishi EA, Agarwal R, Nagar R, Misra A, Kapoor S, Mathur A, Khan MAY, Azizieh F. Shift from a Th1-type response to Th2-type in dengue haemorrhagic fever. Curr Sci 1999;76:63-69. 
7. WHO (1975) Technical Guides for Diagnosis, Treatment,Surveillance, Prevention and Control of dengue Haemorrhagic Fever. World Health Organisation. Geneva

8. Medical Consensus Development Panel, Kementerian Kesihatan Malaysia and Academy of Medicine of Malaysia (1994). Guidelines for the Rational Use Blood and Blood Products.

9. Dengue and Dengue Haemorrhagic fever in the Americas: Guidelines for Prevention and Control 1997. 